\title{
An Optimized Integrin a6-Targeted Magnetic Resonance Probe for Molecular Imaging of Hepatocellular Carcinoma in Mice
}

\author{
Bing-Quan Lin ${ }^{1, *}$ \\ Wen-Biao Zhang ${ }^{2,3, *}$ \\ Jing Zhao ${ }^{3, *}$ \\ Xu-Hui Zhou ${ }^{4, *}$ \\ Yong-Jiang $\mathrm{Li}^{2}$ \\ Jun Deng ${ }^{5}$ \\ Qin Zhao ${ }^{2,3}$ \\ Gui $\mathrm{Fu}^{2,3}$ \\ Chuan-Miao $\mathrm{Xie}^{3}$ \\ Yi-Kai Xu' \\ Guo-Kai Feng ${ }^{2}$
}

'Department of Medical Imaging Center, Nanfang Hospital, Southern Medical

University, Guangzhou City, Guangdong Province, 5105I5, People's Republic of China; ${ }^{2}$ State Key Laboratory of Oncology in South China, Collaborative Innovation Center for Cancer Medicine, Sun Yat-sen University Cancer Center, Guangzhou, 510060, People's Republic of China; ${ }^{3}$ Department of Medical Imaging, Sun Yat-sen University Cancer Center, Guangzhou, 510060, People's Republic of China; ${ }^{4}$ Department of Radiology, The Eighth Affiliated Hospital, Sun Yat-sen University, Shenzhen, 518033, People's Republic of China; ${ }^{5}$ Department of Biological Products, Guangdong Institute for Drug Control, Guangzhou, 510663, People's Republic of China

*These authors contributed equally to this work

Correspondence: Guo-Kai Feng; Yi-Kai $\mathrm{Xu}$

Email fengguok@sysucc.org.cn;

yikaiXu917@gmail.com
Introduction: Integrin $\alpha 6$ is an attractive diagnostic biomarker for molecular imaging of hepatocellular carcinoma (HCC) as it has an extremely high positive rate (approximately 94\%) in clinical early-stage HCC. In this study, based on our previously identified integrin $\alpha 6$-targeted peptide, we developed an optimized integrin $\alpha 6$-targeted magnetic resonance (MR) probe dubbed DOTA(Gd)-ANADYWR for MR imaging of HCC in mice.

Materials and Methods: The longitudinal $\left(\mathrm{R}_{1}\right)$ relaxivity of DOTA(Gd)-ANADYWR was measured on a 3.0 T MR system. The specific tumor enhancement of the agent was investigated in four distinct mouse models, including subcutaneous, orthotopic, genetically engineered and chemically induced HCC mice.

Results: The $\mathrm{R}_{1}$ relaxivity value of DOTA(Gd)-ANADYWR is $5.11 \mathrm{mM}^{-1} \mathrm{~s}^{-1}$ at $3.0 \mathrm{~T}$, which is similar to that of the nonspecific clinical agent Gadoteridol. DOTA(Gd)-ANADYWR generated superior enhanced MR signal in HCC lesions and provided complementary enhancement MR signals to the clinically available hepatobiliary MR contrast agent gadoxetate disodium (GdEOB-DTPA). Importantly, DOTA(Gd)-ANADYWR could efficiently visualize small HCC lesion (approximately $1 \mathrm{~mm}$ ) which was hardly detected by the clinical Gd-EOB-DTPA.

Conclusion: These findings suggest the potential application of this integrin $\alpha 6$-targeted MR probe for the detection of HCC, particularly for small HCC.

Keywords: magnetic resonance, integrin $\alpha 6$, hepatocellular carcinoma, peptide, probe

\section{Introduction}

Liver cancers are the sixth most common cancer and the fourth-leading cause of cancer death worldwide. Hepatocellular carcinoma (HCC) is the dominant histologic type of liver cancers, accounting for $75-85 \%$ of liver cancer cases. ${ }^{1}$ HCC usually develops in patients with cirrhosis related to various etiologies, of which chronic infection by hepatitis $\mathrm{B}$ or $\mathrm{C}$ viruses is the leading cause, followed by alcohol consumption and fatty liver disease associated with metabolic defect. ${ }^{2,3}$ The prognosis of HCC largely depends on the stage at which the tumor is detected. ${ }^{4}$ For patients with early-stage HCC (BCLC stage 0 or A), the 5-year survival rate after curative resection exceeds $60 \%{ }^{5}$ However, for patients with advanced and terminal stage HCC, the median overall survivals are only 11 and $\sim 3$ months, respectively. ${ }^{6}$ Unfortunately, only a minority of patients are diagnosed with resectable HCC, approximately $80 \%$ of HCC patients have advanced unresectable or metastatic at diagnosis. ${ }^{7}$ Therefore, the early diagnosis and treatment are essential to improve the survival rates and prognosis in HCC. 


\section{Graphical abstract}

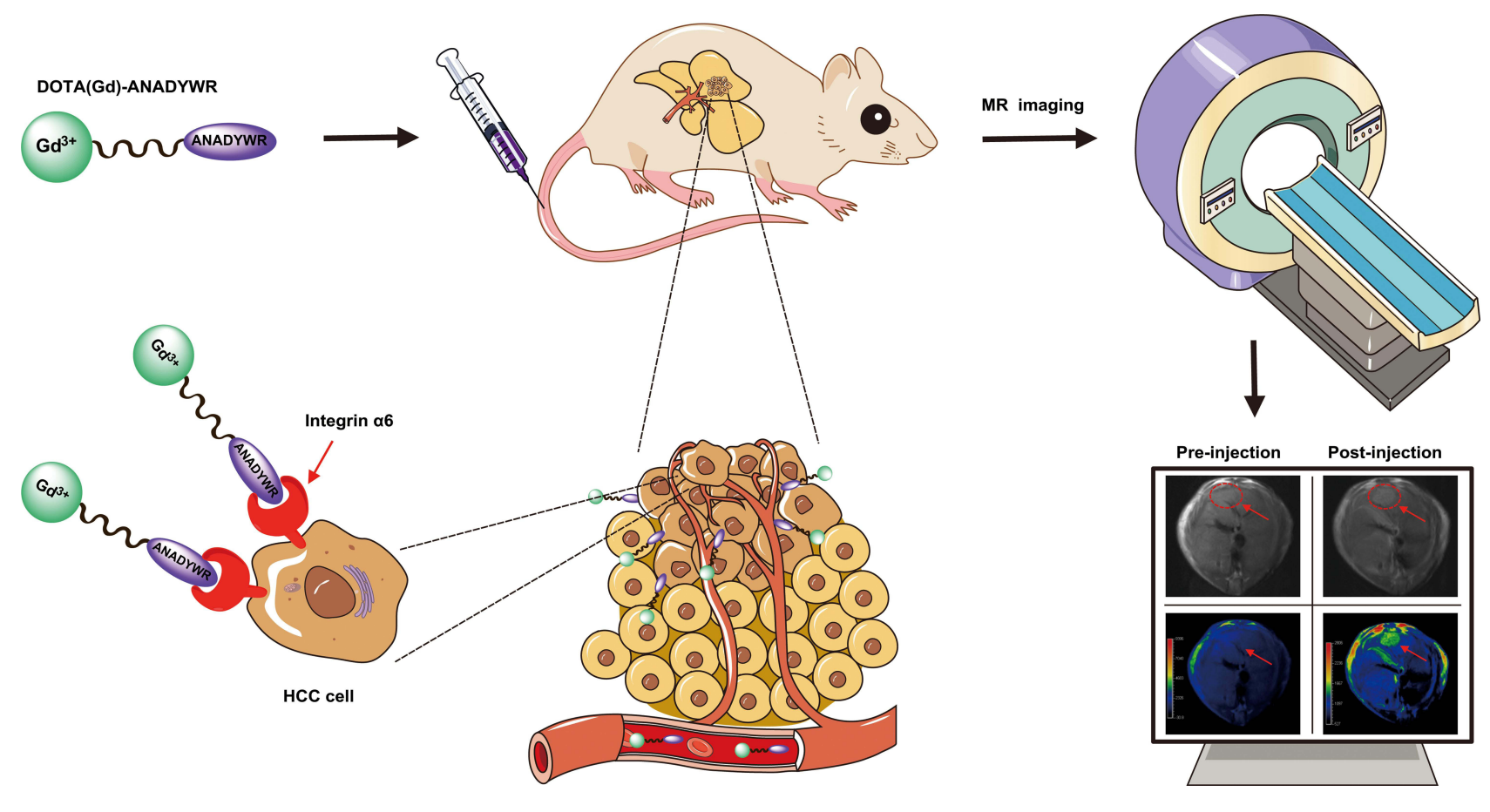

Currently, the diagnosis of HCC is traditionally based on serum biomarkers and/or imaging tests. Alpha fetoprotein (AFP) is the most commonly used of serum biomarkers for HCC diagnosis, but the sensitivity and specificity are only $41-65 \%$ and $80-90 \%{ }^{8,9}$ Traditional imaging modalities that have clinical relevance in HCC diagnosis include ultrasound (US), computed tomography (CT) and magnetic resonance (MR) imaging. ${ }^{10,11}$ US has been extensively used as the preferred imaging modality for screening HCC, with a sensitivity ranging from $40 \%$ to $80 \%$ and a specificity from $82 \%$ to $100 \% .^{10,12-14}$ In a meta-analysis of 15 studies on HCC surveillance in patients with cirrhosis, the pooled sensitivity of US for early-stage HCC detection is only $47 \% .{ }^{14}$ Multi-phase CT or MR imaging has been the standard method for HCC diagnosis, with a sensitivity of $68-91 \%$ and $77-100 \%{ }^{15,16}$ The sensitivities of these anatomic imaging modalities vary according to the size of the liver nodule. ${ }^{17}$ MR imaging offers a higher sensitivity in diagnosing HCC compared to CT, but the sensitivity of MR imaging was only $44 \%$ for small $(1-2 \mathrm{~cm})$ and $15 \%$ for subcentimetric $(<1 \mathrm{~cm})$ focal liver lesions. ${ }^{13,16}$ The above traditional imaging modalities can only acquire anatomical information of HCC, which is difficult to detect small HCC at early stage. Molecular imaging can provide both functional and anatomical information, which may contribute to a higher sensitivity of small HCC. ${ }^{18,19}{ }^{18} \mathrm{~F}$-fluoro-deoxy-glucose is an alternative probe for molecular imaging that can evaluate metabolic activation in vivo, with a sensitivity of $36-70 \%$ for HCC detection. ${ }^{20}$ Gadolinium-ethoxybenzyldiethylenetriamine pentaacetic acid (Gd-EOB-DTPA)enhanced MR imaging has recently been considered as a molecular imaging biomarker of OATP1B3, which has been recommended by many guidelines as an effective method for the detection and characterization of hepatic lesions; however, the per-lesion sensitivity is only $87 \%{ }^{21-23}$ In addition, Gd-EOB-DTPA is not suitable for detecting extrahepatic metastatic HCC due to its characteristic of hepatocyte-specific uptake. ${ }^{24}$ Thus, it is desirable to construct imaging probes specifically targeting hepatic tumors instead of liver parenchyma for early liver cancer diagnosis.

Molecular biomarkers overexpressed on the surface of cancer cells are well-validated targets for molecular imaging of cancer. In our previous study, we screened out a peptide CRWYDDENAC (RWY) by phage display technology specific for nasopharyngeal carcinoma, and further identified that RWY was binding to integrin $\alpha 6$ which was highly expressed in multiple tumors. ${ }^{25}$ Integrins, heterodimeric cell-face receptors composed of $\alpha$ and $\beta$ subunits, 
are closely related to malignant biological behaviors in tumors including migration, invasion, metastasis and angiogenesis via mediating cell adhesion and signal transduction. ${ }^{26-29}$ Integrin $\alpha 6$ subunit, encoded by ITGA6 gene in mammals, can be heterodimerized with either integrin $\beta 1$ subunit or $\beta 4$ subunit to form integrin $\alpha 6 \beta 1$ and $\alpha 6 \beta 4$, binding mostly and specifically to the extracellular matrix laminins. ${ }^{29-31}$ Previous studies indicated that integrin $\alpha 6$ is highly expressed in several tumor tissues and its elevated expression is associated with tumor progression. $^{31-35}$ Similarly, integrin $\alpha 6$ is reported to be highly expressed in HCC, and associated with poor prognosis and worse malignancy. ${ }^{36-39}$ Further, we investigated the transcriptional and translational levels of integrin $\alpha 6$ in HCC by using bioinformatics analysis of GEPIA database (http://gepia.cancer-pku.cn/) and found that integrin $\alpha 6$ expression was significantly higher in early-stage HCC tissues (up to $94 \%$ integrin $\alpha 6$ positive) than in paired adjacent normal tissues. ${ }^{40}$ Therefore, integrin $\alpha 6$ could represent an ideal cancer-specific target for early-stage $\mathrm{HCC}$ diagnosis. On this basis, we have translated the integrin $\alpha 6$ RWY peptide into an MR contrast agent RWY$\mathrm{dL}-(\mathrm{Gd}-\mathrm{DOTA})_{4}$ for MR imaging of $\mathrm{HCC}$ in subcutaneous and chemically induced HCC mice. ${ }^{41}$ Although our previous study has demonstrated the efficacy of integrin a6-targeted RWY-based MR contrast agent for MR imaging of small HCC, the safety and contrast enhancement of RWY-dL-(Gd-DOTA) 4 still need to be improved. Improving the safety and the contrast-enhancement of molecular probe may be achieved by improving its hydrophilicity and the targeting. More recently, we obtained a peptide CRWYDANAC(S5) with higher affinity by alanine scanning, which provides a critical raw material for the second-generation integrin $\alpha 6$-targeted peptide probe. ${ }^{42}$ With high thermodynamic and kinetic stability, macrocyclic Gd(III) chelates exhibit higher biosafety and are increasingly used for synthesis of MR contrast agents. $^{43}$ In this study, we develop an optimized integrin a6-targeted MR contrast agent DOTA(Gd)-ANADYWR based on reverse S5 peptide and macrocyclic Gd(III) chelates for MR imaging of $\mathrm{HCC}$ in subcutaneous, orthotopic, genetically engineered and chemically induced HCC mice.

\section{Materials and Methods}

\section{Cells and Animal Models}

Human HCC cell line HCC-LM3 was purchased from the American Type Culture Collection (ATCC). To facilitate monitoring, luminescent cells HCC-LM3-luciferase cells were generated by stable transfection with luciferase. Cells were grown in RPMI 1640 medium supplemented with $10 \%$ fetal bovine serum, $0.5 \%$ penicillin-streptomycin and incubated at $37{ }^{\circ} \mathrm{C} / 5 \% \mathrm{CO}_{2}$. Female BALB/c nude mice (4 weeks old) and C57BL6 mice (4 weeks old) were purchased from Vita River, Charles River Laboratories China (Beijing, China). All animal experiments were approved by the Institutional Animal Care and Use Committee (IACUC) at Sun Yat-sen University Cancer Center (IACUC approval number L102022020006Y). During the animal experiment, the welfare and treatment of the laboratory animals were followed to "Animal management regulations of China" and "Guangdong experimental animal management regulations", which were published by State Scientific and Technological Commission of The People's Republic of China and People's Government of Guangdong Province, respectively. All the mice models were established according to our previously published methods. ${ }^{40,41,44}$

\section{Preparation of DOTA(Gd)-ANADYWR}

The optimized reverse integrin $\alpha 6$-targeted peptide S5 was synthesized by Chinese Peptide Company (Hangzhou, China) using standard Fmoc-based solid-phase synthesis. The synthesis method of DOTA(Gd)-ANADYWR was based on a previous study from our group and others, with slight modifications. ${ }^{41,43}$ Briefly, $\operatorname{DOTA}(\mathrm{Gd})-$ ANADYWR was synthesized through condensation reaction between carboxyl groups in DOTA and amine groups in reverse S5. The crude product was eluted with a gradient of acetonitrile. Finally, the end product was purified by high-performance liquid chromatography (HPLC) followed by characterized using matrix-assisted laser desorption/ionization time-of-flight (MALDI-TOF) mass spectrometry (Bruker Daltonics, Germany). The longitudinal $\left(\mathrm{R}_{1}\right)$ relaxivity of DOTA(Gd)-ANADYWR was measured on a $3.0 \mathrm{~T}$ MR system (Achieva, 3.0 T, Philips).

\section{In vivo MR Imaging}

MR imaging was performed using a Philips Achieva 3.0 $\mathrm{T}$ system. Mice were anesthetized with 2,2,2-Tribromoethanol $(100 \mu \mathrm{L} / \mathrm{g})$, fixed on the holder and placed into the mouse-imaging coil. At the beginning of scanning, sagittal section images using a localized sequence were obtained to target the HCC lesion. DOTA(Gd)-ANADYWR or control agent was delivered 
by tail vein at a dose of $0.03 \mathrm{mmol}$ of $\mathrm{Gd} / \mathrm{kg}$ for $\mathrm{T} 1$ weighted image in subcutaneous HCC-LM3 tumorbearing mice, orthotopic HCC-LM3-luciferase tumorbearing mice, c-Myc transgenic mice and DEN-induced HCC mice. Either nonspecific clinical agent Gadoteridol or hepatocyte-specific contrast agent Gd-EOB-DTPA (Primovist, Bayer, Germany) was used as a control. On two separate days, mice received either control enhanceagent $(0.03 \mathrm{mmol}$ of $\mathrm{Gd} / \mathrm{kg})$ or DOTA(Gd)-ANADYWR $(0.03 \mathrm{mmol}$ of $\mathrm{Gd} / \mathrm{kg}$ ) and MR imaging, with at least 3 days between scans to ensure most of the gadolinium was cleared. Axial T1-weighted images were acquired by using a turbo spin echo sequence with the following parameters: $\mathrm{TR} / \mathrm{TE}=450 / 15.4 \mathrm{~ms} ; \mathrm{FOV}=4.0 \mathrm{~cm}$; slice thickness $=$ $1 \mathrm{~mm}$; slice spacing $=0.5$; pixel spacing $=0.234 \mathrm{~mm} \times$ $0.234 \mathrm{~mm}$; matrix $=128 \times 128$.

\section{Immunohistochemistry}

At the end of the imaging experiments, all mice were sacrificed by cervical dislocation, and the liver were excised to prepare paraffin sections. Paraffin sections $(3 \mu \mathrm{m})$ were stained with hematoxylin and eosin (H\&E) for routine histologic practice. Immunohistochemical (IHC) staining was performed following conventional procedure, as we reported previously. ${ }^{40}$ In short, paraffin sections were dewaxed into xylene, rehydrated through graded alcohol, and microwaved for antigen retrieval. Blocking to inhibit endogenous peroxidase activity and nonspecific bind, the sections were incubated with an antiintegrin $\alpha 6$ antibody (Abcam, ab181551, 1:150) overnight at $4{ }^{\circ} \mathrm{C}$, followed by an HRP-conjugated polyclonal secondary antibody (1:200) at room temperature for $1 \mathrm{~h}$. Finally, the positive immunoreactivity was visualized by staining with DAB (Zhongshan Jinqiao, ZLI-9017, China) and observed under a microscope (Nikon Eclipse, Japan).

\section{Image Processing and Analysis}

Image analysis was performed using RadiAnt DICOM Viewer (2020.1.1). Contrast-to-noise ratio (CNR) in the HCC was based on the average signal intensity divided by the standard deviation of noise level and calculated according to the previously described formula. ${ }^{41} \mathrm{CNR}-$ Times curves were plotted using the GraphPad Prism 7 software (GraphPad Software Inc., California). Statistical analysis was performed with the SPSS 19.0 software (SPSS, Inc., Chicago, IL, USA). The $P$ values were calculated using two-tailed Student's $t$-test. Significance levels are given as follows: NS $P>0.05, * P<0.05 ; * * P<0.01$; $* * * P<0.001$.

\section{Results}

\section{Synthesis and Characterization}

To improve the contrast-enhancement, we optimized the structure and synthesis of integrin $\alpha 6$-targeted MR probe RWY-dL-(Gd-DOTA $)_{4}$ according to previous reports. ${ }^{42,43}$ Here, we optimized the synthetic procedure of DOTA(Gd)ANADYWR via direct condensation reaction between carboxyl groups in DOTA and amine groups in the reverse S5, thereby rendering the reaction step simpler and the product yields higher. The chemical structure of the optimized integrin $\alpha 6$-targeted MR probe DOTA(Gd)-ANADYWR is shown in Figure 1A. The chemical structure of DOTA(Gd)ANADYWR showed that it has one reverse integrin a6-targeted peptide S5 for tumor targeting, which has a 1.5-fold order higher tumor binding affinity than RWY. ${ }^{42}$ The purity of the synthesized compound was over $95 \%$ (Figure 1B) by HPLC. DOTA(Gd)-ANADYWR was characterized by MALDI-TOF mass spectrometry and had molecular weight in good agreement with the calculated values (Figure 1C). The plots of the $\mathrm{R}_{1}$ versus the concentrations of the contrast agent at 3.0 T are shown in Figure 1D. The $R_{1}$ relaxivity value of DOTA(Gd)-ANADYWR is $5.11 \mathrm{mM}^{-1} \mathrm{~s}^{-1}$ at 3.0 T. DOTA(Gd)-ANADYWR and nonspecific clinical agent Gadoteridol have similar relaxivity values at 3.0 $\mathrm{T}^{45}$

\section{MR Imaging in Subcutaneous, Orthotopic and Genetically HCC Mice}

A flowchart visualizing the MR imaging process is provided in Figure 1E. We first test the contrast enhancement of integrin a6-targeted contrast agent DOTA(Gd)ANADYWR along with the nonspecific clinical agent Gadoteridol for MR imaging in mice bearing subcutaneous HCC-LM3 tumor xenograft. The average tumor size of the subcutaneous HCC mice is $5.99 \pm 1.51 \mathrm{~mm}(\mathrm{n}=3)$. The T2-weighted coronal MR images were obtained to localize the subcutaneous tumor xenografts. Figure $2 \mathrm{~A}$ shows a representative coronal T2-weighted MR image before administration. We quantified the enhancement of the agents in $\mathrm{HCC}$ tissue using the CNR values (Figure 2B). The quantitative CNR values are shown in Table 1. After the injection of DOTA(Gd)-ANADYWR, the T1-weighted signal intensity of HCC rapidly enhanced within the first 5 $\mathrm{min}$, then slowly increased, reached a peak at about $30 \mathrm{~min}$ and remained prominent for at least $50 \mathrm{~min}$. In contrast, 


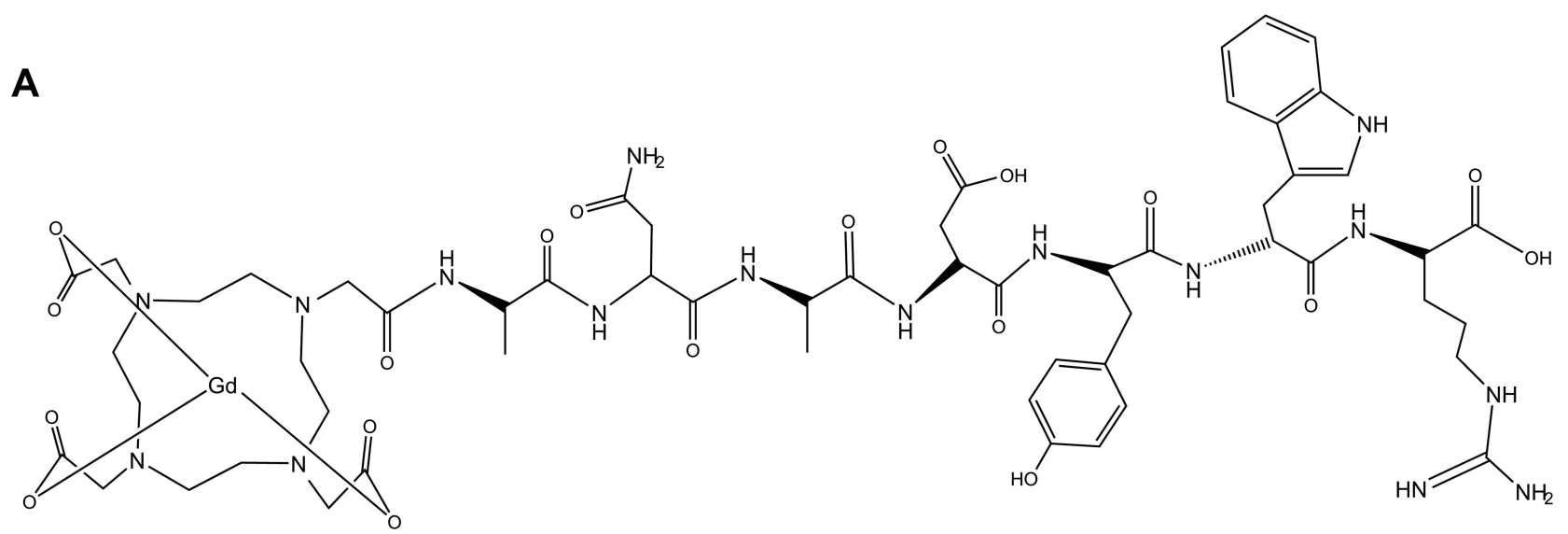

B

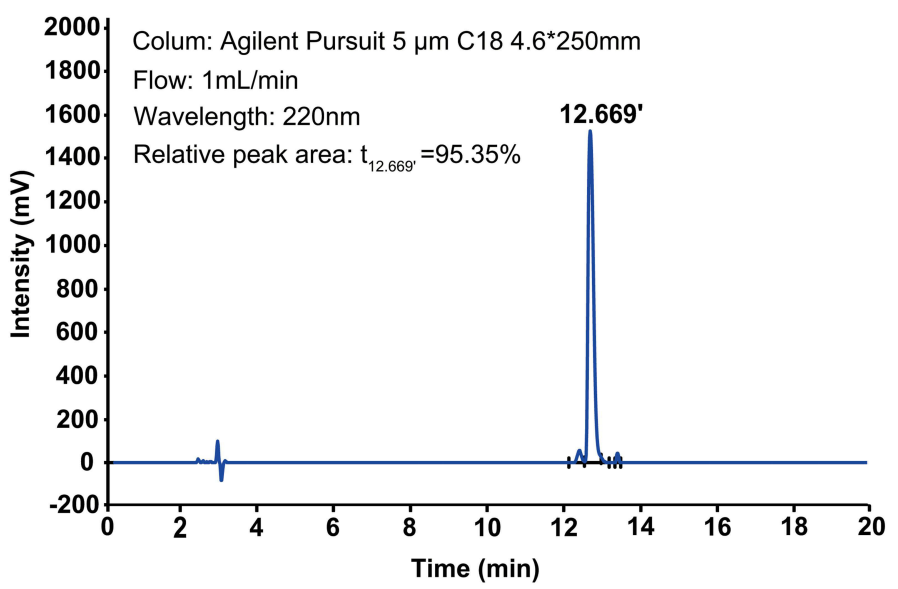

D

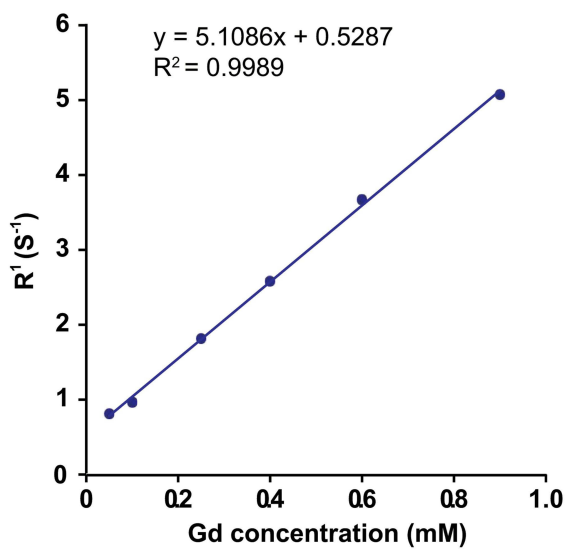

C

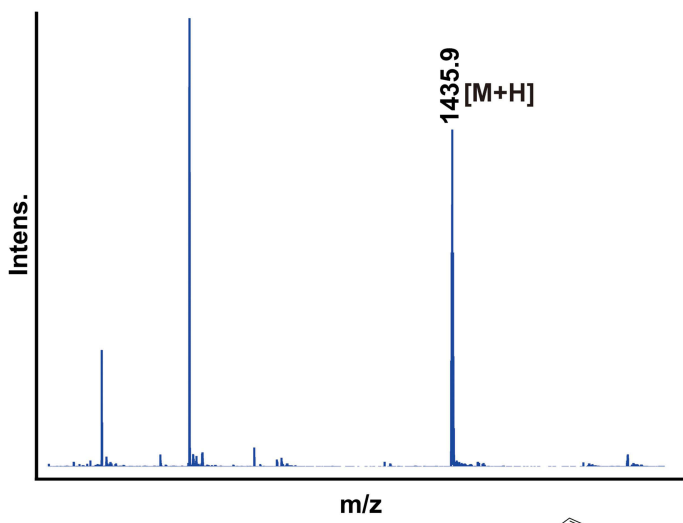

E

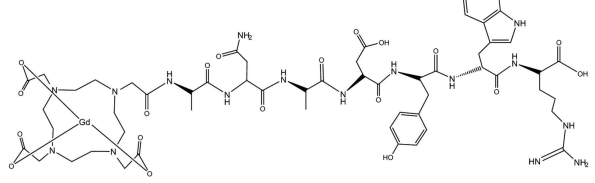

DOTA(Gd)-ANADYWR

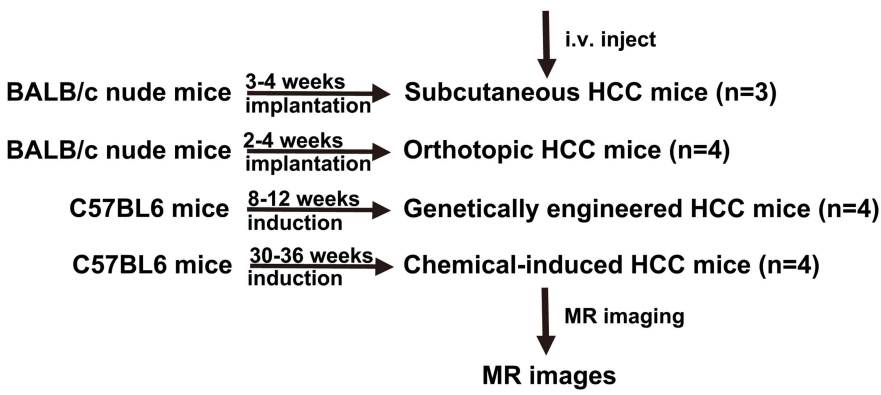

Figure I Chemical structure and characterization of DOTA(Gd)-ANADYWR. (A) chemical structure of DOTA(Gd)-ANADYWR. (B) HPLC analysis of DOTA(Gd)ANADYWR. (C) MALDI-TOF mass spectra of DOTA(Gd)-ANADYWR. (D) Plots of $R_{I}(I / T I)$ versus different gadolinium concentration of DOTA(Gd)-ANADYWR. (E) Flowchart of the study design.

after the injection of Gadoteridol, the signal intensity of HCC rapidly enhanced within the first 5 min, then slowly increased and peaked at about $30 \mathrm{~min}$ followed by a decrease at $40 \mathrm{~min}$. In comparison to Gadoteridol, DOTA(Gd)-ANADYWR produced more significant enhancement in tumor tissue and provided approximately three times improvement in CNR at 50 min pots-injection $(24.76 \pm 6.50$ vs $8.27 \pm 4.23, p=0.03, \mathrm{n}=3)$. Representative T1-weighted MR images obtained before and at different time points after intravenous administration of DOTA(Gd)-ANADYWR or Gadoteridol are shown in Figure 2C. 


\section{A}

\section{Coronal T2-weighted image}

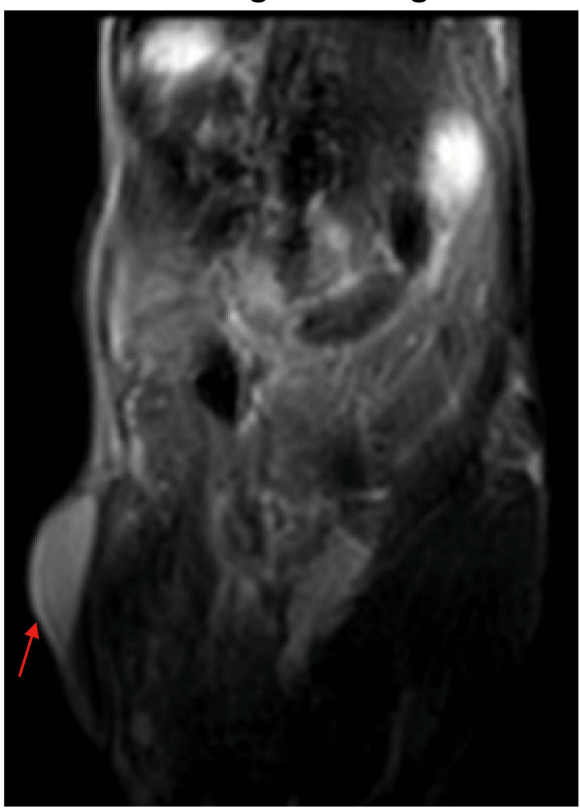

Pre

\section{C}

\section{DOTA(Gd)-ANADYWR}
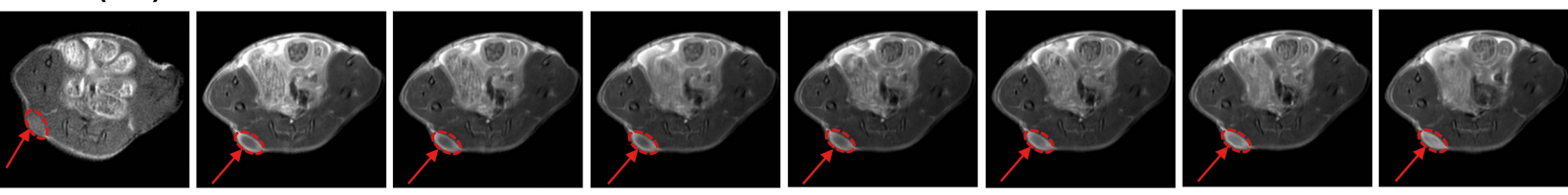

\section{Gadoteridol}

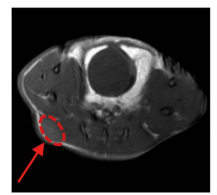

Pre

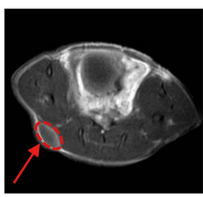

$5 \mathrm{~min}$

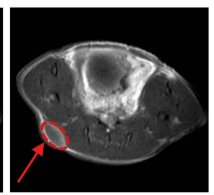

$10 \mathrm{~min}$

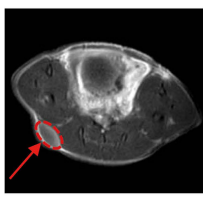

$15 \mathrm{~min}$

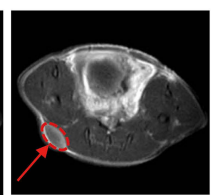

$20 \mathrm{~min}$

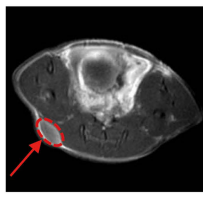

$30 \mathrm{~min}$

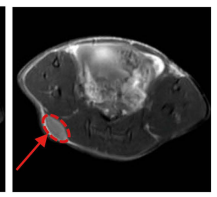

$40 \mathrm{~min}$

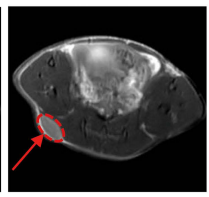

$50 \mathrm{~min}$

Figure 2 MR imaging with DOTA(Gd)-ANADYWR and Gadoteridol in subcutaneous HCC-LM3 tumor-bearing mice. (A) Representative coronal T2-weighted MR image before administration. The red arrowhead indicated the HCC. (B) Contrast-to-noise ratio (CNR) of tumors before and after injection of DOTA(Gd)-ANADYWR or Gadoteridol. Statistical analysis was performed using two-tailed Student's $t$-test. Significance levels are given as: NS $P>0.05$, $* P<0.05$. (C) Representative TI-weighted MR images obtained before and at different time points after intravenous administration of DOTA(Gd)-ANADYWR or Gadoteridol (The red arrowheads indicated the HCC. The red dotted circles delineated the area of interest that used for CNR analysis).

We also tested contrast enhancement of DOTA(Gd)ANADYWR in orthotopic and genetically HCC mice (The average tumor size of the orthotopic and genetically $\mathrm{HCC}$ mice is $3.02 \pm 1.40 \mathrm{~mm}, \mathrm{n}=4$ and $4.52 \pm 1.05 \mathrm{~mm}$, $\mathrm{n}=4$, respectively). Seventeen days after tumor inoculation, the presence of HCC was confirmed by bioluminescence imaging in a representative orthotopic HCC mouse (Figure 3A). T1-weighted MR images of the representative orthotopic HCC mouse were obtained before and $30 \mathrm{~min}$ after
DOTA(Gd)-ANADYWR injection. To improve visibility, MR signals were displayed in rainbow pseudo color (Figure 3B). As shown in Figure 3B, DOTA(Gd)ANADYWR produced significant contrast enhancement in HCC of the orthotopic HCC mouse (The red arrowheads indicate the orthotopic HCC-LM3 tumor xenografts and the red tailless arrowheads indicate the surgical incision sites).

In a 2-months-old c-Myc transgenic mouse, enhanced MR imaging with DOTA(Gd)-ANADYWR was 
Table I The CNR Values of HCC Lesions Obtained Before and at Different Time Points After Intravenous Administration of DOTA(Gd)-ANADYWR or Gadoteridol in Subcutaneous HCC Mice

\begin{tabular}{|c|c|c|c|c|c|c|}
\hline \multirow{3}{*}{$\begin{array}{l}\text { Time } \\
\text { (Min) }\end{array}$} & \multicolumn{6}{|c|}{ CNR Value } \\
\hline & \multicolumn{2}{|c|}{ Mouse I } & \multicolumn{2}{|c|}{ Mouse 2} & \multicolumn{2}{|c|}{ Mouse 3} \\
\hline & $\begin{array}{l}\text { DOTA(Gd)- } \\
\text { ANADYWR }\end{array}$ & Gadoteiridol & $\begin{array}{l}\text { DOTA(Gd)- } \\
\text { ANADYWR }\end{array}$ & Gadoteiridol & $\begin{array}{l}\text { DOTA(Gd)- } \\
\text { ANADYWR }\end{array}$ & Gadoteirido \\
\hline Pre & 1.613 & 3.621 & -0.769 & 0.056 & 0.200 & 0.048 \\
\hline 5 & 12.227 & 5.714 & 15.000 & 10.818 & 18.264 & 11.915 \\
\hline 10 & 16.750 & 7.800 & 17.727 & $|3.7| \mid$ & 21.556 & 7.973 \\
\hline 15 & 21.111 & 8.387 & 15.357 & 11.726 & 20.816 & 11.050 \\
\hline 20 & 21.783 & 11.464 & 16.207 & 12.632 & 18.679 & 9.315 \\
\hline 30 & 26.286 & 12.677 & 22.069 & 16.918 & 20.952 & 10.052 \\
\hline 40 & 28.000 & 13.500 & 22.273 & 15.902 & 18.112 & 10.110 \\
\hline 50 & 30.667 & 3.633 & 25.806 & 11.940 & 17.800 & 9.241 \\
\hline
\end{tabular}

performed to detect the endogenous HCCs, and several enhanced lesions were found in the liver. Figure $4 \mathrm{~A}$ shows the representative T1-weighted MR images obtained before and at different time points after intravenous administration of DOTA(Gd)-ANADYWR. To evaluate the agreement, all MR images were adjusted to consensus window width and window level. To visually observe the enhancement degree of lesions relative to adjacent normal liver tissues, all MR signals were displayed in rainbow pseudo color. We adjusted the window width and level, as appropriate, to make the normal liver tissues represented approximately the same color shade.
A

\section{Overlay}

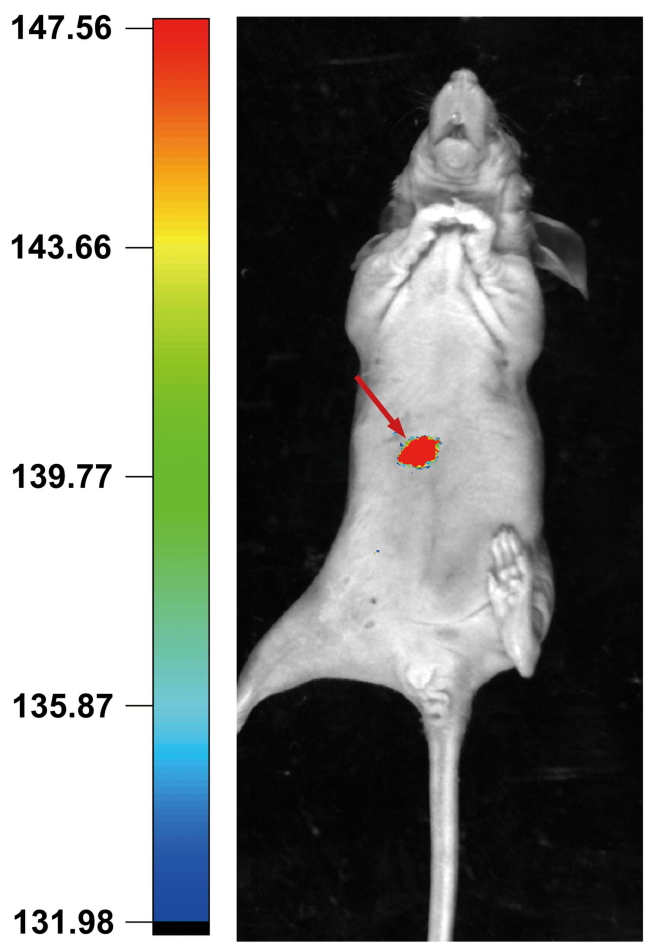

B
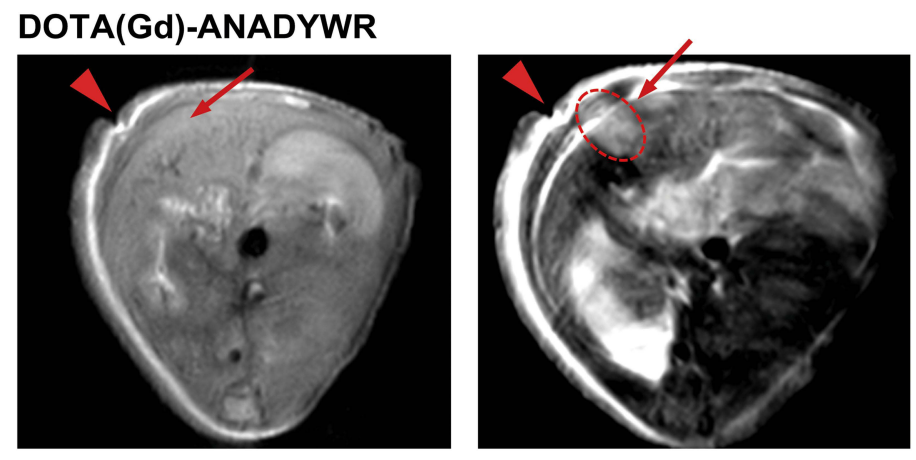

\section{Raibow pseudo color images}

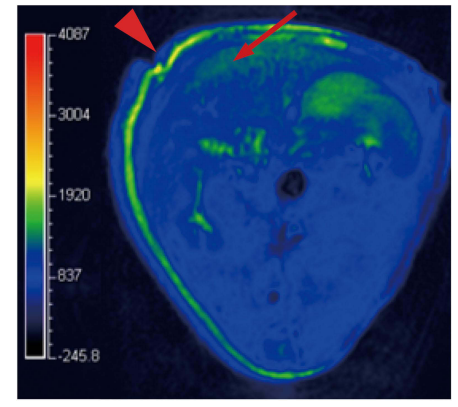

Pre

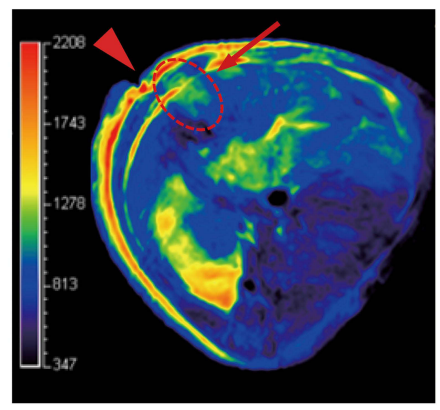

$30 \mathrm{~min}$

Figure 3 MR imaging with DOTA(Gd)-ANADYWR in an orthotopic HCC-LM3-luciferase tumor-bearing mouse. (A) Representative bioluminescence image in a mouse bearing an HCC lesion. (B) Representative TI-weighted MR images and corresponding rainbow pseudo color images obtained before and 30 min after DOTA(Gd)ANADYWR injection in the same mouse (The red arrowheads indicate the orthotopic HCC-LM3 tumor xenografts and the red tailless arrowheads indicate the surgical incision sites. The red dotted circles indicated the visible HCC). 
We also quantified the contrast enhancement between the lesion and the normal liver by using the increased CNR values (Figure 4B). The increased CNR value was calculated as the difference in CNR between lesion and normal liver tissue. As shown in Figure 5B, the increased CNR value rapidly increased within the first $5 \mathrm{~min}$, and then gradually increased over time and remained prominent for at least $50 \mathrm{~min}$. Immunohistochemical staining confirmed the overexpression of integrin $\alpha 6$ in the enhanced lesion (Figure 4C).

\section{Enhanced MR Imaging with DOTA(Gd)-ANADYWR in Detecting Small HCC Lesions}

The diethylnitrosamine (DEN)-induced HCC mouse shares many characteristics with naturally occurring human HCC. ${ }^{40}$ Thus, we assessed the sensitivity of enhanced MR imaging with DOTA(Gd)-ANADYWR in detecting small
HCC lesions by using DEN induced HCC mouse model. The average tumor size of the DEN induced HCC mice is $2.49 \pm 1.12 \mathrm{~mm}, \mathrm{n}=4$. In a mouse nine months after DEN injection, enhanced MR imaging with DOTA(Gd)ANADYWR and then with hepatocellular specific GdEOB-DTPA three days later were performed to detect the small HCC lesions. A small enhanced lesion (red arrowheads, $0.98 \mathrm{~mm}$ in diameter) was found in the liver of the mouse injected with DOTA(Gd)-ANADYWR, but was not found it with Gd-EOB-DTPA (Figure 5A). Gross examination of the liver was conducted after MR imaging. As presented in Figure 5B, gross anatomic examination showed that a white nodule (red arrowhead) was observed in the liver. Immunohistochemical staining confirmed the overexpression of integrin $\alpha 6$ in the white nodule (Figure 5C).

\section{Discussion}

To date, early detection of small HCC remains a great challenge due to the lack of sensitive diagnostic tools.

\section{$A$}

DOTA(Gd)-ANADYWR
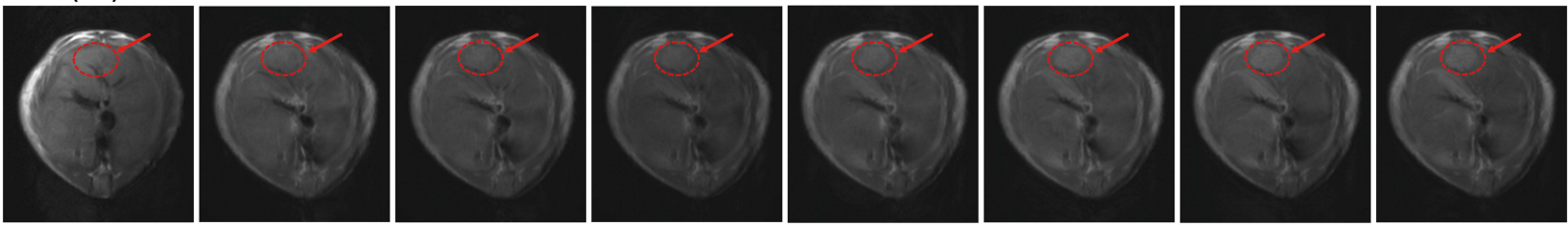

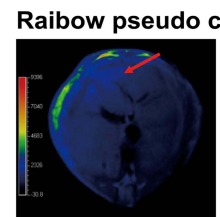

Pre

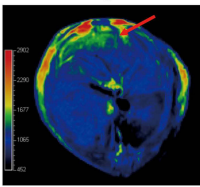

$5 \mathrm{~min}$

B

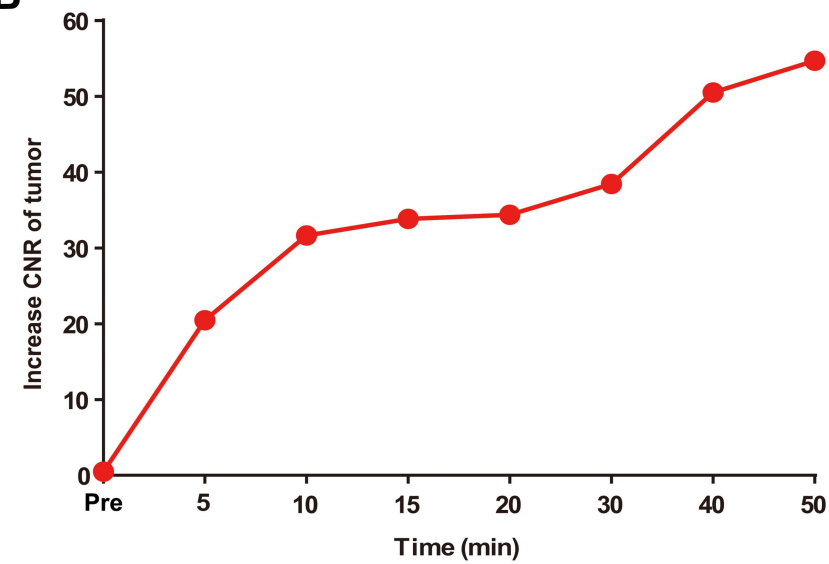

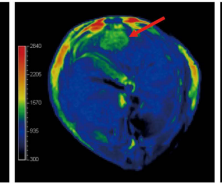

20min

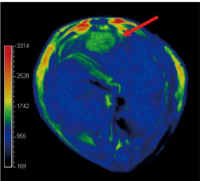

$30 \mathrm{~min}$

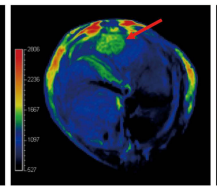

$40 \mathrm{~min}$

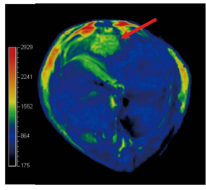

C

IHC

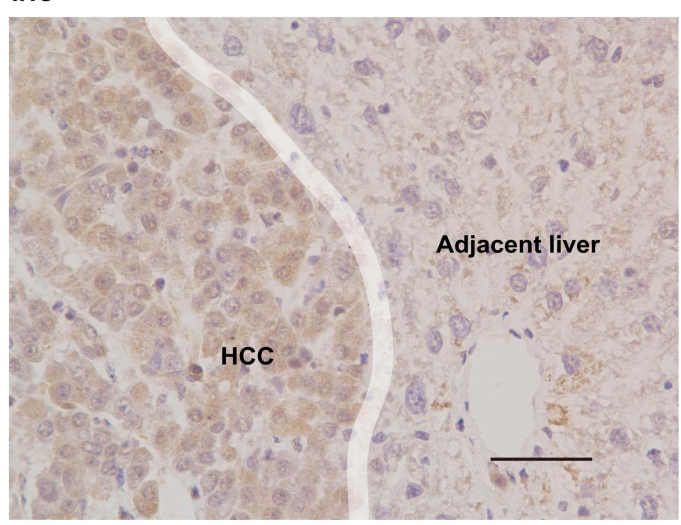

Figure 4 MR imaging with DOTA(Gd)-ANADYWR in a c-Myc transgenic mouse. (A) Representative TI-weighted MR images and corresponding rainbow pseudo color images for a c-Myc transgenic mouse bearing an HCC lesion (The red arrowheads indicated the HCC. The red dotted circles delineated the area of interest that used for CNR analysis). (B) The increase CNR values of tumor before and at different time points after injection of DOTA(Gd)-ANADYWR. (C) Integrin $\alpha 6$ expression was analyzed using immunohistochemistry IHC in tumor tissue (Scale bar, $50 \mu \mathrm{m})$. 


\section{A}

DOTA(Gd)-ANADYWR

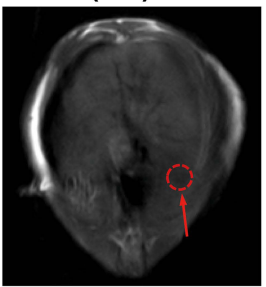

Raibow pseudo color images
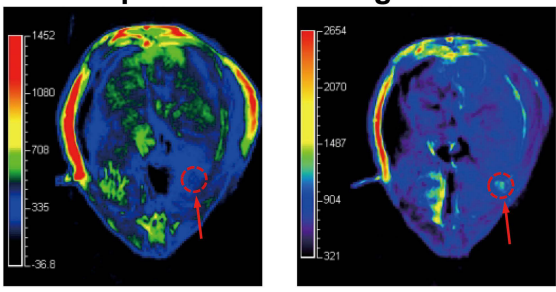

\section{Gd-EOB-DTPA}
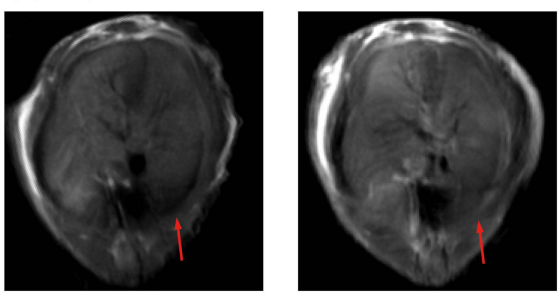

Raibow pseudo color images

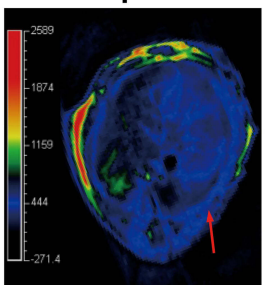

Pre

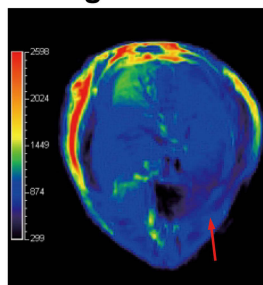

$5 \min$
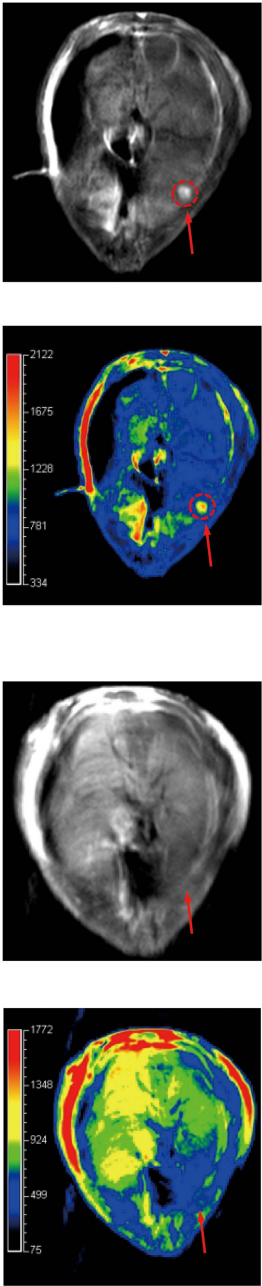

$25 \mathrm{~min}$
B

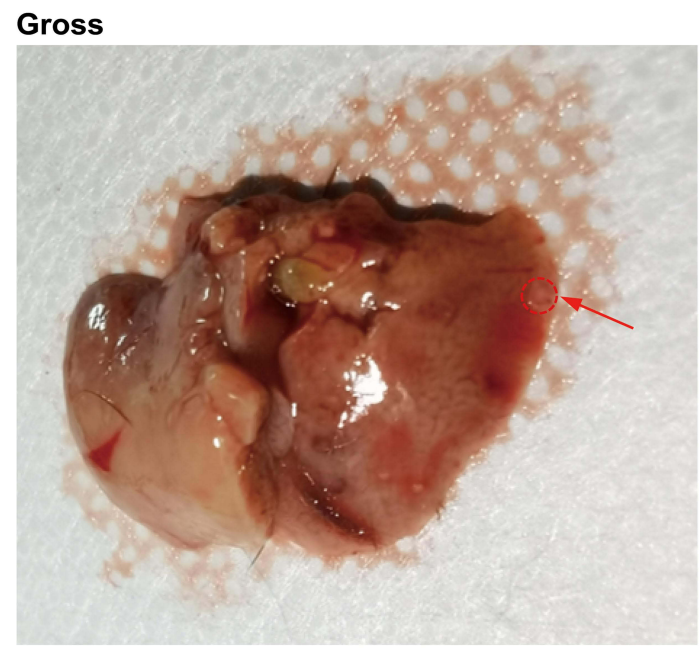

C

IHC

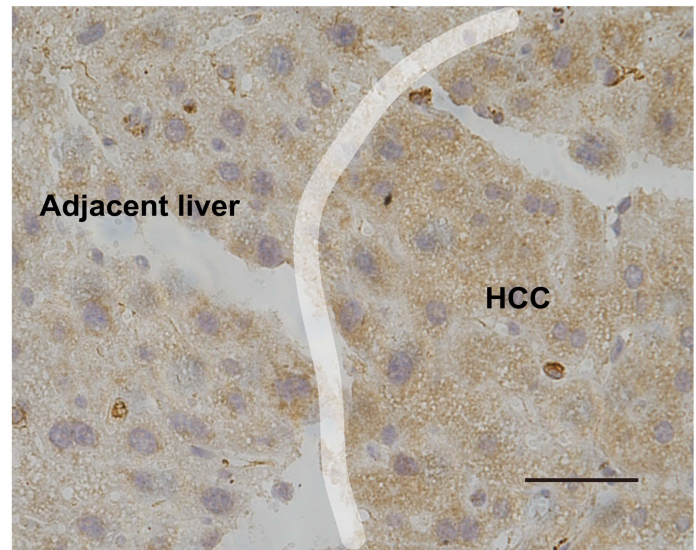

Figure 5 MR imaging with DOTA(Gd)-ANADYWR and Gd-EOB-DTPA in a DEN-induced HCC mouse. (A) Representative MR images and corresponding rainbow pseudo color images obtained before and at 5, 25 min after injection with DOTA(Gd)-ANADYWR or Gd-EOB-DTPA in a DEN-induced HCC mouse bearing an HCC lesion. (B) The corresponding gross photograph of the same lesion shown in $(\mathbf{A})$ (The red arrowheads indicated the HCC. The red dotted circles indicated the visible HCC). (C) Integrin $\alpha 6$ expression was analyzed using immunohistochemistry IHC in tumor tissue (Scale bar, 50 $\mu \mathrm{m}$ ).

The rapid development of molecular imaging has provided powerful tools for the detection of tumor at an early stage. ${ }^{46-50}$ High-resolution imaging device and reliable molecular probe are the pivotal factors that affect the imaging quality of molecular imaging. With the advance of MR technique, the MR imaging quality has greatly improved in recent years. MR imaging provides excellent soft tissue contrast and spatial resolution without ionizing radiation and therefore appears to be the most appropriate for molecular imaging. ${ }^{51,52}$ MR imaging contrast agents can alter the signal intensity in different tissues and thus improve the imaging resolution. ${ }^{53}$ Gadolinium $(\mathrm{Gd})$ complexes are the most commonly used MR imaging contrast agents in the clinic. ${ }^{54}$ However, the present Gd complexes have several disadvantages, including short circulation time, low signal intensity and non-tumor targeting, thus limiting its application in tumor imaging. ${ }^{55,56}$ Therefore, it is desirable to construct a safe and effective MR imaging contrast agent with hepatic tumor specificity for the early HCC detection.

The choice of molecular target is critical for molecular imaging. Molecular imaging target preferably selects proteins that are correlated with tumorigenesis and progression in tumor cells. Here, we selected integrin $\alpha 6$ as a specific target for molecular imaging of $\mathrm{HCC}$ on the basis of our previous studies. ${ }^{40,41}$ The reasons for the choice of integrin $\alpha 6$ as a target can be justified as follows: First, integrin $\alpha 6$ is highly expressed in HCC and its 
elevated expression correlates with the malignancy and poor progression of HCC. ${ }^{36-41}$ In addition, integrin $\alpha 6$ contains a larger N-terminal extracellular domain, which is more amenable to bind molecular probe. ${ }^{57}$ Given the above, integrin $\alpha 6$ can be an ideal target for HCC molecular imaging. However, the most widely studied member of integrins in molecular imaging is integrin $\alpha v \beta 3$, which can be specifically recognized by Arg-Gly-Asp (RGD) sequence. ${ }^{58-63}$ Previous studies of integrin $\alpha 6$ have focused mainly on its role in driving tumorigenesis, and its role in molecular imaging has been scarcely investigated. ${ }^{64-66}$ In our previous study, we firstly demonstrated that integrin $\alpha 6$ can serve as an effective target for molecular imaging in $\mathrm{HCC}^{40}$ As shown in the previous study, integrin $\alpha 6$-targeted PET tracer ${ }^{18} \mathrm{~F}-\mathrm{RWY}$ has a relatively higher sensitivity and specificity than integrin av $\beta 3$-targeted PET tracer ${ }^{18} \mathrm{~F}-3 \mathrm{PRGD}_{2}$ for $\mathrm{HCC}$ detection. ${ }^{40}$ Considering the fact that MR imaging is cheap, widely available and devoid of ionizing radiation, we subsequently validated the effectiveness of integrin $\alpha 6$ for MR imaging of HCC. ${ }^{41}$ Our initial study successfully demonstrated the feasibility for using integrin $\alpha 6$-target MR imaging probe to detect $\mathrm{HCC}$ in mice. Encouragingly, integrin $\alpha 6$-target $\mathrm{MR}$ imaging probe RWY-dL-(Gd-DOTA $)_{4}$ produced superior enhanced MR signal in HCC lesions and provided complementary enhanced MR signal to Gd-EOB-DTPA. ${ }^{41}$ The above results showed great potential for integrin $\alpha 6$-target molecular probe to MR imaging in HCC.

In this study, we successfully developed an optimized MR probe DOTA $(\mathrm{Gd})$-ANADYWR based on optimized integrin a6-targeted S5 peptide and macrocyclic Gd(III) chelates for imaging HCC in four distinct mouse models, including subcutaneous, orthotopic, genetically engineered and chemicalinduced HCC mice. We demonstrated the effectiveness of this optimized MR probe for the detection of HCC in subcutaneous, orthotopic, and genetically engineered mice. In addition, we demonstrated the feasibility of enhanced MR imaging with DOTA(Gd)-ANADYWR in detecting small HCC lesions with superior image quality compared with GdEOB-DTPA by using DEN induced HCC mouse model.

Overall, our findings are in agreement with expectations. As the integrin $\alpha 6$-targeted S5 peptide we selected in a recent study has a higher affinity for integrin $\alpha 6$ than those used previously, DOTA(Gd)-ANADYWR theoretically has superior contrast enhancement than the nonspecific clinical agent Gadoteridol and RWY-dL-(GdDOTA $)_{4}$. Indeed, as displayed in Figure 2, the DOTA(Gd)-
ANADYWR does produce more significant signal enhancement in the HCC lesions than Gadoteridol. Importantly, compared to the earlier targeted agent RWY$\mathrm{dL}-(\mathrm{Gd}-\mathrm{DOTA})_{4}$, the signal intensity generated by DOTA(Gd)-ANADYWR was still slowly increasing after the initial rapid enhanced stage (5 min after injection), until it reached a peak at $30 \mathrm{~min}$ and sustainably prominent for at least 50 min. ${ }^{41}$ The above results may be attributed to a higher accumulation of optimized targeted agent in the tissue of the tumor due to its higher affinity for integrin $\alpha 6$. More importantly, this may be useful to increase the sensibility of target agent for small HCC. As expected, by utilizing the optimized targeted agent DOTA(Gd)ANADYWR, a small HCC $(<1 \mathrm{~mm})$ was significantly intensified in the liver of a DEN induced HCC mouse. However, the hepatocellular specific Gd-EOB-DTPA did not produce visible contrast enhancement in the same lesion (Figure 5).

Nevertheless, there remain several limitations in this study. First, the tumor MR signal intensity reaches a peak at about $30 \mathrm{~min}$, which is likely too long for medical imaging applications. The binding affinity of integrin $\alpha 6$ peptide still needs to be improved. Second, the toxicology, physicochemical and pharmacokinetic properties of the optimized targeted agent remain unknown. Clearly, further research in this area is needed before any clinical application.

\section{Conclusion}

In conclusion, DOTA(Gd)-ANADYWR can serve as an effective MR contrast probe for the detection of HCC in mice. DOTA(Gd)-ANADYWR can produce superior enhanced MR signal in HCC lesion and detect HCC smaller than $1 \mathrm{~mm}$. DOTA(Gd)-ANADYWR exhibits great potential for noninvasive early detection of HCC.

\section{Acknowledgments}

This work was carried out with the support of the Key Project of the National Natural Science Foundation (Grant: 51937010), the National Natural Science Foundation of China (NSFC) (Grant: 81972531, 81871334), the Medical Scientific Research Foundation of Guangdong Province (Grant: A2020155) and the Fundamental Research Funds for the Central Universities (Grant: 19ykpy174). The authenticity of this article has been validated by uploading the key raw data onto the Research Data Deposit public platform (www.researchdata.org.cn), with the approval RDD number as RDDB2021001613. 


\section{Disclosure}

The authors declare that they have no known competing financial interests or personal relationships that could have appeared to influence the work reported in this paper.

\section{References}

1. Bray F, Ferlay J, Soerjomataram I, Siegel RL, Torre LA, Jemal A. Global cancer statistics 2018: GLOBOCAN estimates of incidence and mortality worldwide for 36 cancers in 185 countries. $C A$ Cancer J Clin. 2018;68(6):394-424. doi:10.3322/caac.21492

2. Sanyal AJ, Yoon SK, Lencioni R. The etiology of hepatocellular carcinoma and consequences for treatment. oncologist. 2010;15 (Suppl 4):14-22. doi:10.1634/theoncologist.2010-S4-14

3. Chen WQ, Zheng RS, Baade PD, et al. Cancer Statistics in China, 2015. Ca-Cancer J Clin. 2016;66(2):115-132. doi:10.3322 caac. 21338

4. Zhang Z, Cai M, Bao C, Hu Z, Tian J. Endoscopic Cerenkov luminescence imaging and image-guided tumor resection on hepatocellular carcinoma-bearing mouse models. Nanomedicine. 2019;17:62-70. doi:10.1016/j.nano.2018.12.017

5. Villanueva A. Hepatocellular Carcinoma. $N$ Engl J Med. 2019;380 (15):1450-1462. doi:10.1056/NEJMra1713263

6. Marquardt JU, Thorgeirsson SS. SnapShot: hepatocellular carcinoma. Cancer Cell. 2014;25(4):550. doi:10.1016/j.ccr.2014.04.002

7. Ng KT, Yeung OW, Liu J, et al. Clinical significance and functional role of transmembrane protein 47 (TMEM47) in chemoresistance of hepatocellular carcinoma. Int $J$ Oncol. 2020. doi:10.3892/ ijo.2020.5104

8. Zhao T, Jia L, Li J, et al. Heterogeneities of Site-Specific N-Glycosylation in HCC Tumors With Low and High AFP Concentrations. Front Oncol. 2020;10:496. doi:10.3389/fonc.20 20.00496

9. Gupta S, Bent S, Kohlwes J. Test characteristics of alpha-fetoprotein for detecting hepatocellular carcinoma in patients with hepatitis C. A systematic review and critical analysis. Ann Intern Med. 2003;139(1):46-50. doi:10.7326/0003-4819-139-1-200307010-00012

10. Jiang HY, Chen J, Xia CC, Cao LK, Duan T, Song B. Noninvasive imaging of hepatocellular carcinoma: from diagnosis to prognosis. World j Gastroenterol. 2018;24(22):2348-2362. doi:10.3748/wjg. v24.i22.2348

11. Francica G, Borzio M. Status of, and strategies for improving, adherence to HCC screening and surveillance. $J$ Hepatocellular Carcinoma. 2019;6:131-141. doi:10.2147/JHC.S159269

12. Bolondi L. Screening for hepatocellular carcinoma in cirrhosis. $J$ Hepatol. 2003;39(6):1076-1084. doi:10.1016/S0168-8278(03) 00349-0

13. Yang JD, Kim WR. Surveillance for hepatocellular carcinoma in patients with cirrhosis. Clin Gastroenterol Hepatol. 2012;10 (1):16-21. doi:10.1016/j.cgh.2011.06.004

14. Tzartzeva K, Obi J, Rich NE, et al. Surveillance Imaging and Alpha Fetoprotein for Early Detection of Hepatocellular Carcinoma in Patients With Cirrhosis: a Meta-analysis. Gastroenterology. 2018;154(6):1706-1718. doi:10.1053/j.gastro.2018.01.064

15. Sangiovanni A, Manini MA, Iavarone M, et al. The diagnostic and economic impact of contrast imaging techniques in the diagnosis of small hepatocellular carcinoma in cirrhosis. Gut. 2010;59 (5):638-644. doi:10.1136/gut.2009.187286

16. Hennedige T, Venkatesh SK. Advances in computed tomography and magnetic resonance imaging of hepatocellular carcinoma. World j Gastroenterol. 2016;22(1):205-220. doi:10.3748/wjg.v22.i1.205

17. Ronot M, Clift AK, Vilgrain V, Frilling A. Functional imaging in liver tumours. $J$ Hepatol. 2016;65(5):1017-1030. doi:10.1016/j. jhep.2016.06.024
18. Weissleder R. Molecular imaging: exploring the next frontier. Radiology. 1999;212(3):609-614. doi:10.1148/radiology.212.3. r99se18609

19. $\mathrm{Hu} \mathrm{Z}$, Chi C, Liu M, et al. Nanoparticle-mediated radiopharmaceutical-excited fluorescence molecular imaging allows precise image-guided tumor-removal surgery. Nanomedicine. 2017;13(4):1323-1331. doi:10.1016/j.nano.2017.01.005

20. Lee SM, Kim HS, Lee S, Lee JW. Emerging role of (18) F-fluorodeoxyglucose positron emission tomography for guiding management of hepatocellular carcinoma. World $j$ Gastroenterol. 2019;25(11):1289-1306. doi:10.3748/wjg.v25.i11.1289

21. Lee YJ, Lee JM, Lee JS, et al. Hepatocellular carcinoma: diagnostic performance of multidetector CT and MR imaging-a systematic review and meta-analysis. Radiology. 2015;275(1):97-109. doi:10.1148/radiol.14140690

22. Kumada T, Toyoda H, Yasuda S, et al. Impact of the introduction of direct-acting anti-viral drugs on hepatocarcinogenesis: a prospective serial follow-up MRI study. Aliment Pharmacol Ther. 2020;52 (2):359-370. doi:10.1111/apt.15825

23. Kudo M. Gd-EOB-DTPA-MRI Could Predict WNT/beta-Catenin Mutation and Resistance to Immune Checkpoint Inhibitor Therapy in Hepatocellular Carcinoma. Liver Cancer. 2020;9(5):479-490. doi:10.1159/000509554

24. Xue S, Yang H, Qiao J, et al. Protein MRI contrast agent with unprecedented metal selectivity and sensitivity for liver cancer imaging. Proc Natl Acad Sci U S A. 2015;112(21):6607-6612. doi:10.1073/pnas.1423021112

25. Feng GK, Zhang MQ, Wang HX. et al. Identification of an Integrin a6-Targeted Peptide for Nasopharyngeal Carcinoma-Specific Nanotherapeutics. Adv Therapeutics;2019. 1900018. doi:10.1002/ adtp. 201900018

26. Hynes RO. Integrins: bidirectional, allosteric signaling machines. Cell. 2002;110(6):673-687. doi:10.1016/S0092-8674 (02)00971-6

27. Cabodi S, Del Pilar Camacho-Leal M, Di Stefano P, Defilippi P. Integrin signalling adaptors: not only figurants in the cancer story. Nat Rev Cancer. 2010;10(12):858-870. doi:10.1038/nrc2967

28. Becchetti A, Arcangeli A. Integrins and ion channels in cell migration: implications for neuronal development, wound healing and metastatic spread. Adv Exp Med Biol. 2010;674:107-123.

29. Takada Y, Ye X, Simon S. The integrins. Genome Biol. 2007;8 (5):215. doi:10.1186/gb-2007-8-5-215

30. Kariya Y, Kariya Y, Gu J. Roles of Integrin alpha6beta4 Glycosylation in Cancer. Cancers. 2017;9(7):79. doi:10.3390/ cancers 9070079

31. Brooks DL, Schwab LP, Krutilina R, et al. ITGA6 is directly regulated by hypoxia-inducible factors and enriches for cancer stem cell activity and invasion in metastatic breast cancer models. Mol Cancer. 2016;15:26. doi:10.1186/s12943-016-0510-x

32. Beaulieu JF. Integrin alpha6beta4 in colorectal cancer. World J Gastrointest Pathophysiol. 2010;1(1):3-11. doi:10.4291/wjgp.v1. i1.3

33. Lathia JD, Gallagher J, Heddleston JM, et al. Integrin alpha 6 regulates glioblastoma stem cells. Cell Stem Cell. 2010;6(5):421-432. doi:10.1016/j.stem.2010.02.018

34. Noh TW, Soung YH, Kim HI, et al. Effect of \{beta 4 integrin knockdown by RNA interference in anaplastic thyroid carcinoma. Anticancer Res. 2010;30(11):4485-4492.

35. Yang XH, Richardson AL, Torres-Arzayus MI, et al. CD151 accelerates breast cancer by regulating alpha 6 integrin function, signaling, and molecular organization. Cancer Res. 2008;68(9):3204-3213. doi:10.1158/0008-5472.CAN-07-2949

36. Begum NA, Mori M, Matsumata T, Takenaka K, Sugimachi K, Barnard GF. Differential display and integrin alpha 6 messenger RNA overexpression in hepatocellular carcinoma. Hepatology. 1995;22(5):1447-1455. doi:10.1002/hep.1840220518 
37. Ke AW, Shi GM, Zhou J, et al. CD151 amplifies signaling by integrin alpha6beta1 to PI3K and induces the epithelial-mesenchymal transition in HCC cells. Gastroenterology. 2011;140(5):1629-1641. doi:10.1053/j.gastro.2011.02.008

38. Ozaki I, Yamamoto K, Mizuta T, et al. Differential expression of laminin receptors in human hepatocellular carcinoma. Gut. 1998;43 (6):837-842. doi:10.1136/gut.43.6.837

39. Torimura T, Ueno T, Kin M, et al. Coordinated expression of integrin alpha6beta1 and laminin in hepatocellular carcinoma. Hum Pathol. 1997;28(10):1131-1138. doi:10.1016/S0046-8177(97)90250-1

40. Feng GK, Ye JC, Zhang WG, et al. Integrin alpha6 targeted positron emission tomography imaging of hepatocellular carcinoma in mouse models. J Control Release. 2019;310:11-21. doi:10.1016/j.jconrel. 2019.08.003

41. Zhang Y, Zhao J, Cai J, et al. Integrin alpha6-Targeted Magnetic Resonance Imaging of Hepatocellular Carcinoma in Mice. Mol Imaging Biol. 2020;22(4):864-872. doi:10.1007/s11307-019-01437-z

42. Mei Y, Li YH, Yang XC, et al. An optimized integrin alpha6-targeted peptide for positron emission tomography/magnetic resonance imaging of pancreatic cancer and its precancerous lesion. Clin Transl Med. 2020;10(4):e157. doi:10.1002/ctm2.157

43. Ayat NR, Qin JC, Cheng H, et al. Optimization of ZD2 Peptide Targeted Gd(HP-DO3A) for Detection and Risk-Stratification of Prostate Cancer with MRI. ACS Med Chem Lett. 2018;9 (7):730-735. doi:10.1021/acsmedchemlett.8b00172

44. Mei Y, Zhou C, Liang CY, et al. A method to establish a c-Myc transgenic mouse model of hepatocellular carcinoma. MethodsX. 2020;7:100921. doi:10.1016/j.mex.2020.100921

45. Shen Y, Goerner FL, Snyder C, et al. T1 relaxivities of gadolinium-based magnetic resonance contrast agents in human whole blood at 1.5, 3, and 7 T. Invest Radiol. 2015;50(5):330-338. doi:10.1097/RLI.0000000000000132

46. Garcia Ribeiro RS, Belderbos S, Danhier P, et al. Targeting tumor cells and neovascularization using RGD-functionalized magnetoliposomes. Int $J$ Nanomedicine. 2019;14:5911-5924. doi:10.2147/IJN.S214041

47. Hu Z, Chen WH, Tian J, Cheng Z. NIRF nanoprobes for cancer molecular imaging: approaching clinic. Trends Mol Med. 2020;26 (5):469-482. doi:10.1016/j.molmed.2020.02.003

48. $\mathrm{Hu} \mathrm{Z}$, Zhao $\mathrm{M}, \mathrm{Qu} \mathrm{Y}$, et al. In vivo 3-dimensional radiopharmaceutical-excited fluorescence tomography. J Nuclear Med. 2017;58(1):169-174. doi:10.2967/jnumed.116.180596

49. Liu H, Yang X, Song T, et al. Multispectral hybrid Cerenkov luminescence tomography based on the finite element SPn method. J Biomed Opt. 2015;20(8):86007. doi:10.1117/1.JBO.20.8.086007

50. Song T, Liu X, Qu Y, et al. A Novel Endoscopic Cerenkov Luminescence Imaging System for Intraoperative Surgical Navigation. Mol Imaging. 2015;14(8):443-449. doi:10.2310/7290.2015.00018

51. Akam EA, Abston E, Rotile NJ, et al. Improving the reactivity of hydrazine-bearing MRI probes for in vivo imaging of lung fibrogenesis. Chem Sci. 2020;11(1):224-231. doi:10.1039/C9SC04821A

52. Nardi-Schreiber A, Gamliel A, Harris T, et al. Biochemical phosphates observed using hyperpolarized (31)P in physiological aqueous solutions. Nat Commun. 2017;8(1):341. doi:10.1038/s41467-01700364-3

Journal of Hepatocellular Carcinoma

\section{Publish your work in this journal}

The Journal of Hepatocellular Carcinoma is an international, peerreviewed, open access journal that offers a platform for the dissemination and study of clinical, translational and basic research findings in this rapidly developing field. Development in areas including, but not limited to, epidemiology, vaccination, hepatitis therapy, pathology
53. Zhou Z, Deng H, Yang W, et al. Early stratification of radiotherapy response by activatable inflammation magnetic resonance imaging. Nat Commun. 2020;11(1):3032.

54. Sani Usman M, Hussein MZ, Fakurazi S, Masarudin MJ, Ahmad Saad FF. Gadolinium-Doped Gallic Acid-Zinc/Aluminium-Layered Double Hydroxide/Gold Theranostic Nanoparticles for a Bimodal Magnetic Resonance Imaging and Drug Delivery System. Nanomaterials. 2017;7(9):244. doi:10.3390/nano7090244

55. Gao A, Teng Y, Seyiti P, et al. Using Omniscan-Loaded Nanoparticles as a Tumor-Targeted MRI Contrast Agent in Oral Squamous Cell Carcinoma by Gelatinase-Stimuli Strategy. Nanoscale Res Lett. 2019;14(1):395. doi:10.1186/s11671-019-3214-5

56. Wahsner J, Gale EM, Rodriguez-Rodriguez A, Caravan P. Chemistry of MRI Contrast Agents: current Challenges and New Frontiers. Chem Rev. 2019;119(2):957-1057. doi:10.1021/acs.chemrev.8b00363

57. Longmate WM, Dipersio CM. Integrin Regulation of Epidermal Functions in Wounds. Adv Wound Care. 2014;3(3):229-246. doi:10.1089/wound.2013.0516

58. Wang J, Zhao H, Zhou Z, et al. MR/SPECT Imaging Guided Photothermal Therapy of Tumor-Targeting $\mathrm{Fe} @ \mathrm{Fe} 3 \mathrm{O} 4$ Nanoparticles in Vivo with Low Mononuclear Phagocyte Uptake. ACS Appl Mater Interfaces. 2016;8(31):19872-19882. doi:10.1021/ acsami.6b04639

59. Wang T, Hou Y, Bu B, et al. Timely Visualization of the Collaterals Formed during Acute Ischemic Stroke with $\mathrm{Fe} 3$ O4 Nanoparticle-based MR Imaging Probe. Small. 2018;14(23):e1800573.

60. Boucher M, Geffroy F, Preveral S, et al. Genetically tailored magnetosomes used as MRI probe for molecular imaging of brain tumor. Biomaterials. 2017;121:167-178. doi:10.1016/j.biomaterials.2016. 12.013

61. Zhang C, Jugold M, Woenne EC, et al. Specific targeting of tumor angiogenesis by RGD-conjugated ultrasmall superparamagnetic iron oxide particles using a clinical 1.5-T magnetic resonance scanner. Cancer Res. 2007;67(4):1555-1562. doi:10.1158/0008-5472.CAN06-1668

62. Chen K, Chen X. Integrin targeted delivery of chemotherapeutics. Theranostics. 2011;1:189-200. doi:10.7150/thno/v01p0189

63. Chen H, Niu G, Wu H, Chen X. Clinical Application of Radiolabeled RGD Peptides for PET Imaging of Integrin alphavbeta3. Theranostics. 2016;6(1):78-92. doi:10.7150/thno.13242

64. Yao H, Price TT, Cantelli G, et al. Leukaemia hijacks a neural mechanism to invade the central nervous system. Nature. 2018;560 (7716):55-60. doi:10.1038/s41586-018-0342-5

65. Nikolopoulos SN, Blaikie P, Yoshioka T, Guo W, Giancotti FG. Integrin beta 4 signaling promotes tumor angiogenesis. Cancer Cell. 2004;6(5):471-483. doi:10.1016/j.ccr.2004.09.029

66. Shaw LM, Rabinovitz I, Wang HH, Toker A, Mercurio AM. Activation of phosphoinositide 3-OH kinase by the alpha6beta4 integrin promotes carcinoma invasion. Cell. 1997;91(7):949-960. doi:10.1016/S0092-8674(00)80486-9 and molecular tumor classification and prognostication are all considered for publication. The manuscript management system is completely online and includes a very quick and fair peer-review system, which is all easy to use. Visit http://www.dovepress.com/ testimonials.php to read real quotes from published authors. 